\title{
A gas chromatographic analysis method development and validation for determination of common plasticizers in delayed release tablet dosage forms
}

\begin{abstract}
A simple and efficient Gas Chromatography (GC) analysis method was developed to identify and quantify plasticizers commonly used with polymers present in delayed release tablets. The plasticizers investigated included Dibutyl Sebacate (DBS), Tributyl Phosphate (TBP) and Tributyl Acetyl Citrate (TBAC). The Gas Chromatography (GC) analysis method employs a common DB-1 GC column $(30 \mathrm{mx} 320 \mu \mathrm{m} ; 3.0 \mu \mathrm{m}$ film). The method was shown to be specific and linear $\left(\mathrm{r}^{2}=0.998-1.000\right)$. Both accuracy and precision were established across the analytical range $(0.8-2.0 \mathrm{mg} / \mathrm{mL})$. Method applicability was demonstrated by analyzing currently marketed DR tablets from different manufacturers.
\end{abstract}

Keywords: gas chromatography, plasticizers, dibutyl sebacate, tributyl phosphate, tributyl acetyl citrate, delayed release tablets, high-performance liquid chromatography, tributyl phosphate, polymeric films, drug release, polymers, physico-chemical properties, validation, standard calibration, coating films, data processing
Volume 8 Issue 6 - 2019

\section{Prasad Panzade, Jessie Wang, Yon Wang}

Department of R\&D AD, Apotex Inc, Canada

Correspondence: Prasad Panzade, Department of R\&D AD, Apotex Inc, I 50 Signet Drive, Toronto, Ontario M9L IT9, Canada, Email prasad_panzadel320@yahoo.com, ppanzade@apotex.com

Received: November 28, 2019 | Published: December 17 2019
Abbreviations: DR, delayed release; TBAC, tributyl acetyl citrate; DBS, dibutyl sebacate; TBP, tributyl phosphate; GC, gas chromatography; HPLC, high-performance liquid chromatography; QL, quantitation limit; ICH, International conference on harmonization guidelines; LOQ, limit of quantitation

\section{Introduction}

Various polymers $(\mathrm{MW}>100,000)$ are widely used materials to retard the drug release from controlled release pharmaceutical preparations. Polymers present in film coated delayed release (DR) tablets are typically used together with plasticizers such as Dibutyl Sebacate (DBS), Tributyl Phosphate (TBP) and Tributyl Acetyl Citrate (TBAC). Plasticizers are recognized as a critical aspect for drug delivery. They are relatively small lubricating molecules (MW 200400) that can modify physico-chemical properties and process ability of the polymers, improve the mechanical properties of a polymer matrix, and increase the workability, flexibility or extensibility of the polymer. Also, plasticizers are generally used to modify the thermal properties, water absorption behavior, and adhesive properties of polymeric films. The concentration of plasticizer affects the strength of coating films, overall integrity of drug products and drug release characteristics in therapeutic pharmaceutical dosage forms. ${ }^{1,2}$

The quality of coating directly impacts the drug release profile, especially for controlled release drug products. Therefore, the consistency of coating should be carefully maintained to ensure consistent drug release and reaching the required therapeutic concentration within a pre-determined time period at specific $\mathrm{pH}$. For example, some drugs can only be released in colon at $\mathrm{pH}$ about 7.2. The intra luminal $\mathrm{pH}$ is rapidly changing from acidic $(\mathrm{pH} 1-2)$ in the stomach to $\mathrm{pH}$ about 6 in the duodenum. The $\mathrm{pH}$ gradually increases in the small intestine from $\mathrm{pH} 6$ to about $\mathrm{pH} 7.4$ in the terminal ileum.
The $\mathrm{pH}$ drops to 5.7 in the caecum, but again gradually increases, reaching pH 6.7 in the rectum. ${ }^{3}$ The drug release is controlled mainly by the polymer and its characteristics which strongly depend on the presence and amount of the plasticizer. Therefore, controlling the concentration of the plasticizer in film coated DR tablets is an essential part of the overall quality assessment and control. The objective of this study was to develop a simple and reliable Gas Chromatography (GC) analysis method to identify and quantify plasticizers commonly used with polymers present in delayed release tablets. The plasticizers investigated in this study included Dibutyl Sebacate (DBS), Tributyl Phosphate (TBP) and Tributyl Acetyl Citrate (TBAC).

\section{Materials and methods}

\section{Reagents, chemicals and samples}

High-Performance Liquid Chromatography (HPLC) grade Methanol used as sample solvent was purchased from EMD Millipore Corporation, Dibutyl Sebacate (DBS) was obtained from Vertellus Specialties Inc., Dibutyl Phthalate (DBP) and Tributyl 2-Acetylcitrate (TBAC) were obtained from Sigma-Aldrich. Currently marketed DR tablets from two different manufactures (Samples A, B and C, respectively) and one DR tablets sample from Apotex (Sample D) were used for the GC testing to quantify the level of plasticizers.

\section{Apparatus, software and chromatography}

An Agilent Technologies 7890A Gas Chromatography (GC) system, equipped with an FID detector, an Agilent Technologies injector of $7683 \mathrm{~B}$ and DB-1 column $(30 \mathrm{mx} 320 \mu \mathrm{m} ; 3.0 \mu \mathrm{m}$ film), was used for the experiments. The Gas Chromatography (GC) system, data acquisition and processing were controlled using Empower computer program. Helium was used as carrier gas and make-up gas. Detection temperature: $300^{\circ} \mathrm{C}$; split ratio: $20: 1$; split flow: $70 \mathrm{~mL} / \mathrm{min}$; oven 
program: $200^{\circ} \mathrm{C}, 5 \mathrm{~min}, 10^{\circ} \mathrm{C} / \mathrm{min} ; 280^{\circ} \mathrm{C}, 7 \mathrm{~min}$. Injection volume: $1.0 \mu \mathrm{L}$. The data collection, standard calibration and data processing used Empower 3.

\section{Standard solution preparation}

$40 \mathrm{mg}$ each of DBS, DBP and TBAC were weighted into a $50 \mathrm{~mL}$ of volumetric flask containing about $40 \mathrm{~mL}$ of methanol. Dilution to volume with methanol and mixing yields the standard solution containing $0.8 \mathrm{mg} / \mathrm{mL}$ of each plasticizer. The GC chromatogram of standard solution is presented in Figure 1.

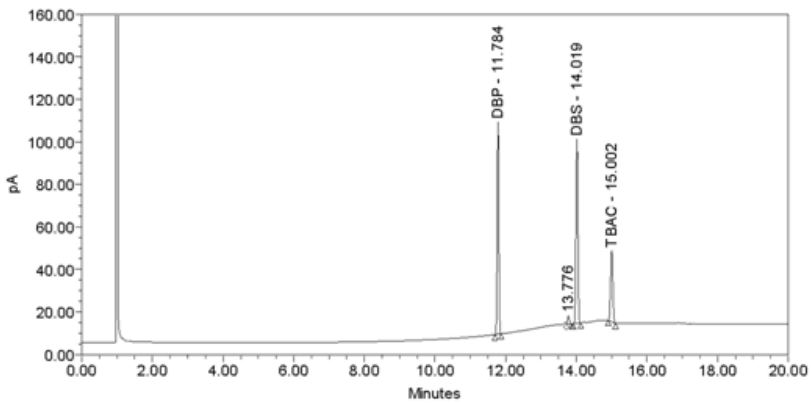

Figure I GC chromatogram of standard solution containing $0.8 \mathrm{mg} / \mathrm{mL}$ each of DBS, DBP and TBAC.

\section{Sample Solution Preparation}

One tablet was placed into a $25 \mathrm{~mL}$ volumetric flask containing 20 $\mathrm{mL}$ of methanol, the flask was vortexed to remove coating completely, methanol was added to the volume, the content was mixed filtered through a Whatman PTFE $(0.45 \mu \mathrm{m}, 25 \mathrm{~mm})$, first $5 \mathrm{~mL}$ were discarded before collecting the sample solution for injection.

\section{Calculation}

The amount of a plasticizer (PS) in the tablet is determined using the equation below:

$$
P S(m g / t a b)=A s p l / A s t d x \text { Wstd / Vstd } x \text { Vspl }
$$

Where,

Aspl and Astd - peak area in the sample and standard chromatogram, respectively,

Wstd - standard weight (mg),

Vstd and Vspl - volume of standard and sample solution, respectively

\section{Method validation}

\section{Limit of quantification (LOQ) ${ }^{4}$}

The working standard concentration is $0.8 \mathrm{mg} / \mathrm{mL}$ each of DBS, DBP and TBAC, the quantitation limit (QL) concentration was $10 \%$ of the working standard concentration i.e. $0.08 \mathrm{mg} / \mathrm{mL}$ each of DBS, DBP and TBAC. The \%RSD of six consecutive injections for System Precision at QL was $1.1 \%, 1.4 \%$ and $10.4 \%$, for DBS, DBP and TBAC, respectively. The results met criteria of method validation as per International Conference on Harmonization Guideline (ICH) of $\%$ RSD is not more than $15.0 \%$ for Residual Solvents. Therefore, this method can quantify the plasticizers at $10 \%$ level of working standard concentration $(0.08 \mathrm{mg} / \mathrm{mL})$.

\section{Linearity ${ }^{4}$}

The linearity plots were generated for the peak area of DBS, DBP and TBAC against its respective concentrations (linearity range: 0.08 - $2.0 \mathrm{mg} / \mathrm{mL}$ ). The plots are presented in Figure 2. The $\mathrm{R}^{2}$ values for DBS, DBP and TBAC were $1.000,1.000$ and 0.998 , respectively. Peak response and its concentration between $0.08 \mathrm{mg} / \mathrm{mL}$ and $2.0 \mathrm{mg} /$ $\mathrm{mL}$ it met criteria as per International Conference on Harmonization Guideline of $\mathrm{R}^{2}$ is not less than 0.98 .
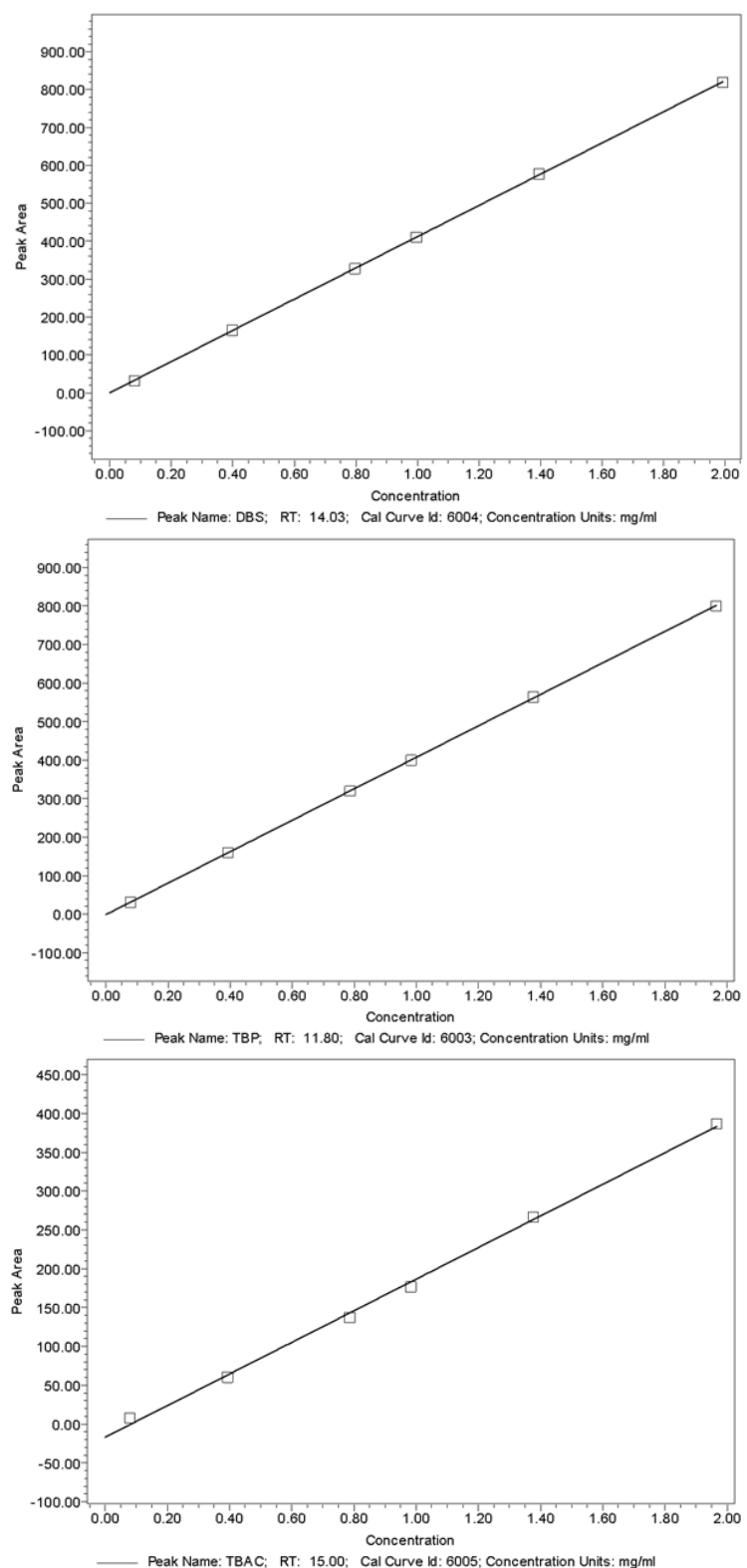

Figure 2 Linearity plots of DBS (top), DBP (middle) and TBAC (bottom).

\section{Accuracy and method precision ${ }^{5}$}

Accuracy was evaluated using pre-mixed coating excipients except DBS, DBP or TBAC, then spiked at $10 \%, 100 \%$ and $150 \%$ levels for each PS. Method Precision on six samples was performed at 100\% level $(0.8 \mathrm{mg} / \mathrm{mL})$. The \%found of DBS, DBP and TBAC in unspiked sample was zero. The results of recovery of DBS, DBP and TBAC at 
different levels are summarized in Table 1. The results met criteria of method validation as per International Conference on Harmonization Guideline (ICH) of \%Recovery within $80 \%-120 \%$ and \%RSD is not more than $15 \%$ for Residual Solvents. It can be concluded that the developed Gas Chromatography (GC) analysis method generates accurate and precise results for determination of the concentration of all three studied plasticizers: DBS, DBP and TBAC.

Table I Recovery and precision study results for DBS, DBP and TBAC

\begin{tabular}{|c|c|c|c|c|c|c|}
\hline \multirow{2}{*}{ Study/Level } & \multicolumn{2}{|l|}{ DBS } & \multicolumn{2}{|l|}{ DBP } & \multicolumn{2}{|l|}{ TBAC } \\
\hline & \%Recovery & \%RSD & \%Recovery & \%RSD & \%Recovery & \%RSD \\
\hline Accuracy QL-I & 99 & I & 99 & 1 & 95 & 2.1 \\
\hline Accuracy QL-2 & 97 & & 97 & & 97 & \\
\hline Accuracy QL-3 & 98 & & 98 & & 99 & \\
\hline Precision - sample I & 99 & I.I & 99 & 0.8 & 105 & 1.2 \\
\hline Precision - sample 2 & 100 & & 100 & & 108 & \\
\hline Precision - sample 3 & 100 & & 100 & & 108 & \\
\hline Precision - sample 4 & 101 & & 101 & & 108 & \\
\hline Precision - sample 5 & 98 & & 99 & & 106 & \\
\hline Precision - sample 6 & 99 & & 100 & & 108 & \\
\hline Accuracy $150 \%-1$ & 99 & 1.5 & 100 & I & 108 & 1.4 \\
\hline Accuracy $150 \%-2$ & 101 & & 101 & & 110 & \\
\hline Accuracy $150 \%-3$ & 98 & & 99 & & 107 & \\
\hline
\end{tabular}

\section{Specificity ${ }^{4}$}

The chromatogram of the unspiked accuracy sample, containing all excipients except DBS or DBP or TBAC, showed no interference with the peaks of interest. Also, there was no interference from the active ingredient in the sample chromatograms. This method is therefore specific to DBS, DBP and TBAC. A Whatman PTFE $(0.45 \mu \mathrm{m}, 25 \mathrm{~mm})$ filter was used for sample preparation. This filter is suitable as evidenced by the filter study performed for the $1^{\text {st }}, 2^{\text {nd }}, 3^{\text {rd }}$, $5^{\text {th }}$ and $6^{\text {th }} \mathrm{mL}$ of the filtrations. The recovery of DBS, DBP and TBAC was $100 \%$ for all the fractions.

\section{Statistical analysis}

Since this method was validated with Accuracy, Method Precision met criteria as per International Conference on Harmonization Guideline ( $\mathrm{ICH})$, therefore the test results considered to be accurate. The same sample solutions were re-injected and confirmed the very close results.

\section{Testing results of marketed DR tablets for plasticizers}

Using the Gas Chromatography (GC) analysis method described above, three samples of the same drug product (DR tablets), manufactured and marketed by two different suppliers, were acquired and tested for plasticizers along with the analogous Apotex DR tablets. The results are summarized in Table 2. The data show that the manufactures of the marketed products (samples A, B and C) are using different plasticizers while Apotex employs DBS, similar to the manufacturer of Sample A. Thus, the developed Gas Chromatography
(GC) analysis method was confirmed to be suitable for determination of the content of various plasticizers in DR tablets from different sources.

Table 2 Content of plasticizers in marketed DR tablets

\begin{tabular}{llll}
\hline & DBS mg/tablet & DBP mg/tablet & TBAC mg/tablet \\
\hline Sample A & 6.2 & - & - \\
Sample B & - & 8.5 & - \\
Sample C & - & - & 13.9 \\
Sample D & 20.2 & - & - \\
\hline
\end{tabular}

\section{Discussion}

This Gas Chromatography (GC) analysis method was developed to identify and quantify plasticizers (Dibutyl Sebacate, Tributyl Phosphate and Tributyl Acetyl Citrate) commonly used with polymers present in delayed release tablets, which pharmaceutical need to have a good control of drug release time, to get the best medication function.

The method was shown to be specific, linear, accurate and precise. The International Conference on Harmonization Guideline (ICH) criteria for analytical method validation were met and proves the testing results are accuracy and repeatable.

\section{Conclusions}

The method is simple in implementation. Specifically, sample preparation step is quick and easy to perform owing to the easiness 
of extraction of plasticizers which are freely soluble in methanol allowing for complete extraction into the organic solvent and separation from the polymeric matrix. The Gas Chromatography (GC) analysis method compares with the High-Performance Liquid Chromatography (HPLC) method for determination of a number of plasticizers in pharmaceutical dosage forms available in the literature. ${ }^{6}$ Specifically, the limit of quantitation (LOQ) of $0.08 \mathrm{mg} /$ $\mathrm{mL}$ determined for the developed Gas Chromatography (GC) method is about two times lower than the LOQ of $0.5 \mathrm{mM} / \mathrm{L}$ (corresponding to $0.157 \mathrm{mg} / \mathrm{mL}$ ) reported in the literature for dibutyl sebacate. Gas Chromatography (GC) method is more sensitive to it. The limitation of this method is that for molecular weight more than 400, e.g. carnauba wax will be difficult, maybe need per treatment (reaction) before the testing.

\section{Acknowledgments}

None.

\section{Conflicts of interest}

The author declares that there are no conflicts of interest.

\section{Funding}

None.

\section{References}

1. Somwanshi SB, Dolas RT, Wagh VD, et al. Pharmaceutically Used Plasticizers: A Review. European Journal of Biomedical and Pharmaceutical Sciences. 2016;3(2):277-285.

2. EI-Gendy NA, Pharmaceutical Plasticizers for Drug Delivery Systems. Curr. Drug Deliv. 2012;9(2):148-163.

3. Fallingborg J. Intraluminal $\mathrm{pH}$ of the human gastrointestinal tract. Dan Med Bull. 1999;46(3):183-196.

4. Galina Holloway, Dibutyl Sebacate, USP-41 NF36. 5324.

5. International Conference on Harmonization Guideline for Residual Solvents. 1997.

6. Bodmeier R, Paeratakul O. Determination of Plasticizers Commonly Used In Pharmaceutical Dosage Forms by High Performance Liquid Chromatography. J Liq Chrom., 1991;14(2):365-375. 